\title{
فلس ساماني نادر ضرب بخارا سنة 358هـ باسم الأمير منصور بن نوح
}

\section{A Rare Samanid fils Struck in Bukhara in 358AH.Bearing the Name of Prince Al-Mansour Bin Noah}

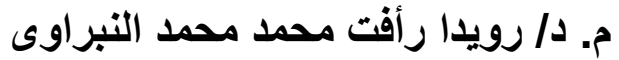

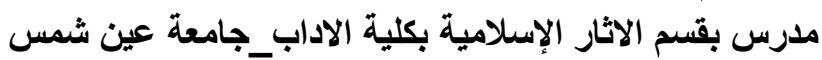

\section{Dr. Rowida Raafat Mohamed Mohamed Elnabrawy \\ Lecturer in Islamic department, faculty of Arts, Ain Shams university. \\ Rowida_khaled@hotmail.com}

\begin{abstract}
يتناول هذا البحث دراسة ونشر لفلس سامانى نادر ضرب بخارا سنة 358هـ بإسم الأمير منصور بن نوح (350-

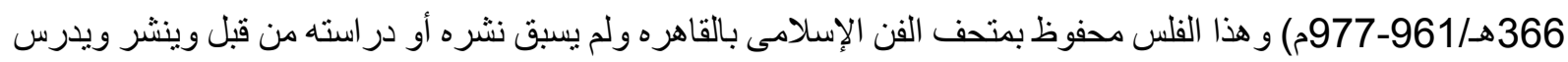
في هذا البحث لأول مرة. وقد تم وصف الثكل العام لهذا الفلس وتحليل كتاباته ، ويحمل هذا الفلس كتابات مركزية وأخرى فى هامثين بالوجه وكتابات مركزية وأخرى هامشية واخر بالظهر وتقع كتابات مركز الوجه فى أربعة أسطر، يشتمل السطر الأول على اسم"فائق" و هو ابو الحسن ابن عبداله فائق الذى عرف بفائق الخاصة لأنه كان من خاصة مربي الأمير منصور بن نوح كما أنه هو

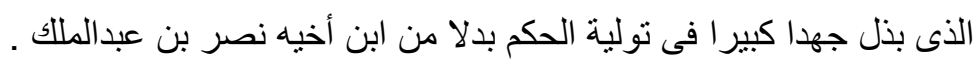

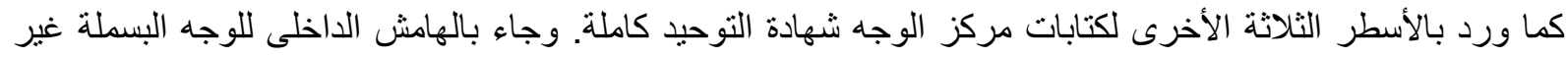

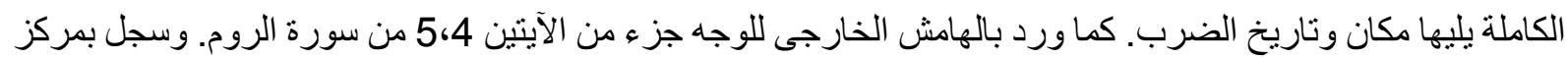
الظهر كتابات فى خمسة أسطر تشتمل على كلمة "له" يليها الرسالة المحمدية مختصرة فى سطرين ثم إسم منصور بن بن نوح

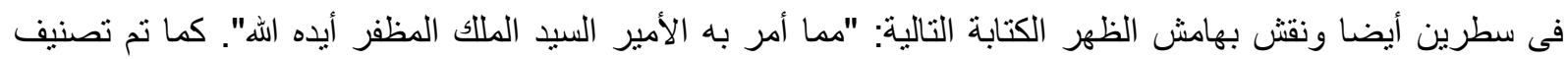
الفلوس السامانية التىى تحمل إسم الأمير منصور بن نوح و المضروبة فى بخار اسنة 358هـ وسجل عليها إسم فائق بكتابات مركز الوجه الى خمسة طرز وتم وصف شكلها وتحليل كتاباتها والمقارنة بينها كما تم أيضا مقارنتها بالفلس موضوع البحث وتم التوصل الى أن طر از الفلس موضوع البحث يختلف عن هذه الطرز الخمسة ويمثل طر ازا جديدا لم ينشر مثيل له. ويعد إضافة جديدة لفلوس الأمير منصور بن نوح المضروبة فى بخار اسنة 358هـ وتحمل إسم فائق.

\section{الكثمات المفتاحية: \\ فلس، ساماني، منصور، بخار ا، فائق}

\begin{abstract}
:
This research deals with studying and publishing a rare Samanid fals minted in Bukhara issued in 358 A.H (=969 AD) by the prince Mansour bin Noah (350-366AH / 961-977 AD). This fils is preserved in the Museum of Islamic Art in Cairo and it has never been published or studied before and it is being published and studied in this research for the first time. The study includes detailed description and epigraphs analysis.

The front numismatic text of this fils enclouds central epigraphs consists of four lines; the first line has the name of "Faeq"فائق Abu Al-Hassan Ibn Abdullah Faeq “The special one", because he was among the breeders of Prince Mansour bin Noah, as he was the one who made a great effort in supporting him to assuming power rather than his nephew "Nasr bin Abdul Malik". The other three lines of the central epigraphs includes a complete Tawhid declaration.
\end{abstract}


The central epigraphs surrounded by two Marginalepigraphs; the internal one enclouds incomplete Basmala besides the date and location of the minting, while the external epigraphs have surah Ar-Rūm (verses 4 \& 5).

The back numismatic text has five lines includes the word god "الهُ" followed by the Muhammadiyah message, shortened in two lines, then the name of Mansour bin Noah in two lines. The central epigraphs surrounded by a Marginalthat has the following text: was ordered by the Prince Sayyid al-Malikar al-Mudhafar, may God support him.

Samanid fulus that bearing the name of Prince Mansour bin Noah, which was minted in Bukhara in 358 A.H and has the name of "Faeq" on the front center was categorized into five styles and were descried based on shapes and epigraphs but this Samanid fals is considered a new style that has not been published before and it is considered a new addition to the coins of Prince Mansour bin Noah that minted in Bukhara in the year 358 AH and bears Faeq's name.

\section{Key words:}

Fils, Samanid, Mansour, Bukhara, Fae'k

يقتني متحف الفن الإسلامي بالقاهرة فلس ساماني نادر (1) ضرب بخار اسنة 358هـ بإسم الأمير منصور بن نوح2 (350366هـ/1961-977م)( لوحة رقم 1) لم يسبق نشره أو در استه من قبل ويُنشر ويدرس في هذا البحث لأول مرة .

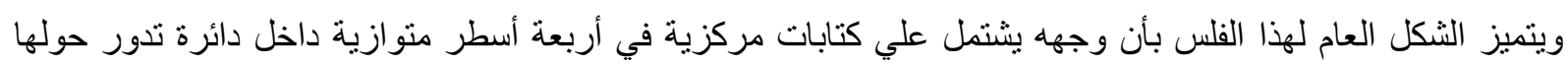
كتابات في هامشين لا يفصلهما أية دوائر، وتحيط بكتابات الهامش الخارجي من الخارج دائرة، أما الظهر فيشتمل علي فئي كتابات مركزية في خمسة أسطر داخل دائرة من حبيبات غير منماسة تدور حولها كتابات في هامش واحد فقط تحيط بها

$$
\text { من الخارج دائرة. }
$$

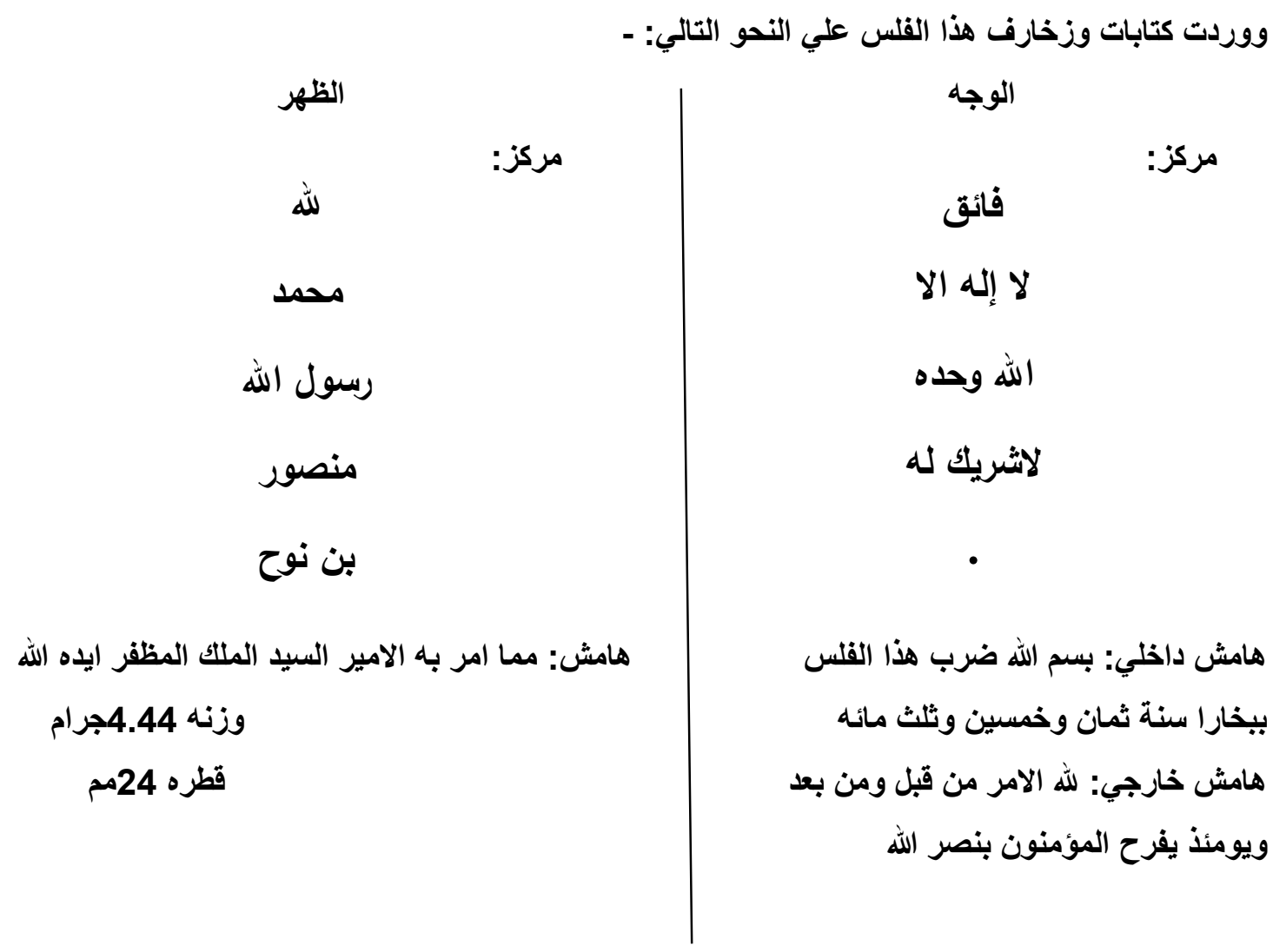


يوجد أعلي كتابات مركز الوجه بالسطر الأول إسم " فائق" وهو أبو الحسن بن عبد الله فائق الذي عرف بفائق الخاصة لأنه

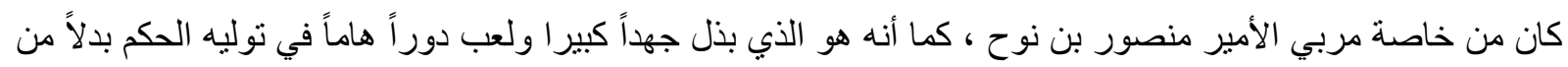

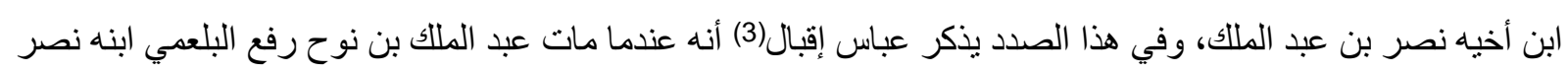

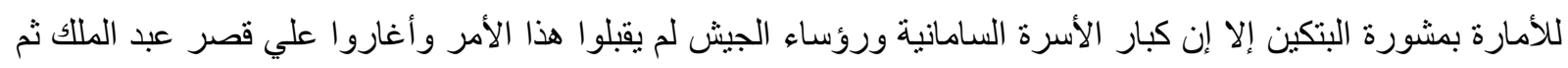

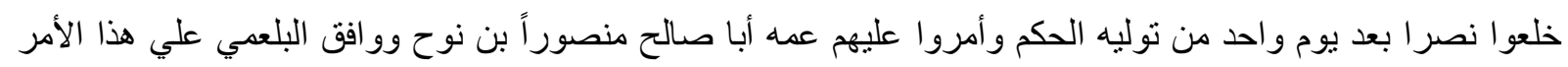
ولذلك ظل في منصب الوزارة، وكان أكثر الناس سعياً لإبلاغ المنصور للإمارة فهو الأمير الحسن بن عبد اله فائق الذي في كان من الغلمان الروميين أصلاً، وكان منذ طفولته في خدمة منصور بن نوح ومن خاصة مربيه ولهذ ولهذا سمي فائق الخاصة. ونقش في الأسطر الثناثة الأخيرة وهي من السطر الثاني حتى السطر الرابع بكتابات مركز الوجه شهادة التوحيد كاملة

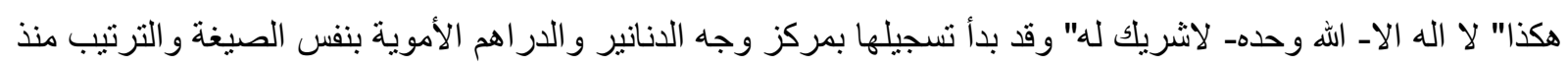

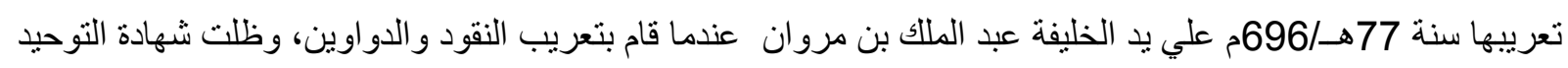

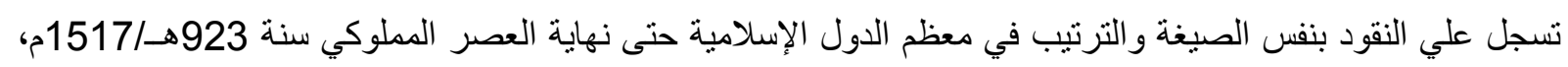

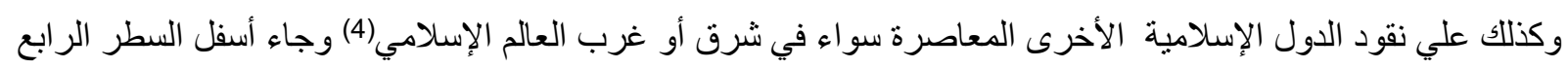

و الأخير بكتابات مركز الوجه نقطة صغيرة مطموسة.

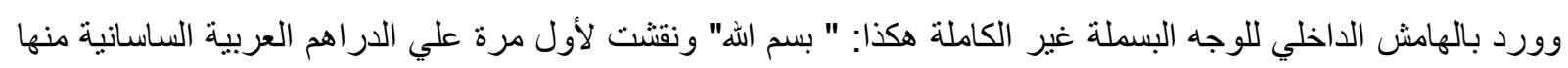

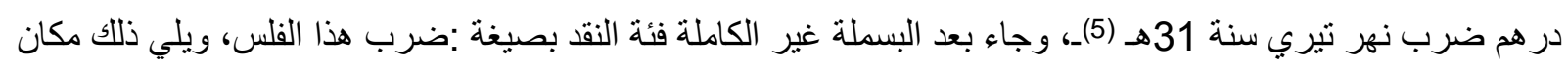

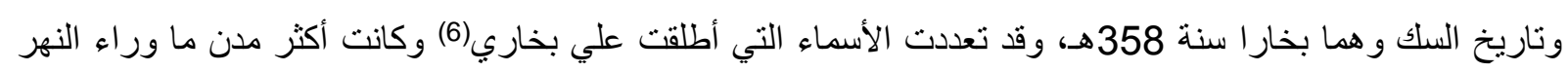
ازدهار اوكانت بخاري عاصمة للسامانيين ملوك الشرق منذ سنة 279هـ/892م حينما اتخذها الأمير إسماعيل بن أحمد وكد

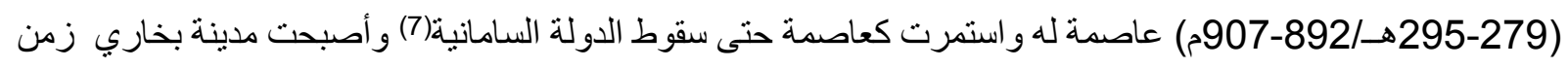
الأمير المذكور أعظم مدن العالم الإسلامي إزدهار|(8) وضربت في بخارا النقود الذهبية والفضية والنحاسية وتميزت

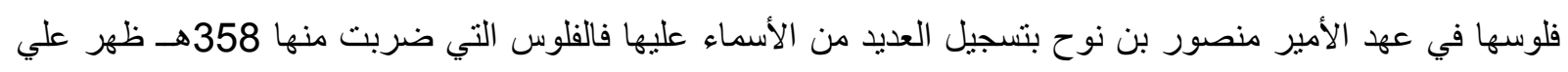

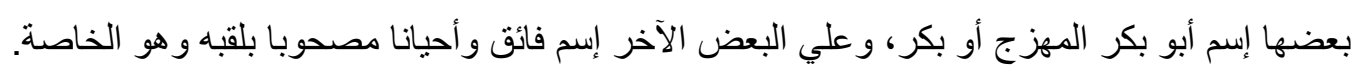

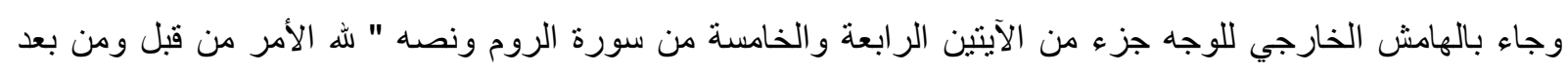
ويومئذ يفرح المؤمنون بنصر اله " وقد نزلت هذه السورة عندما إنتصر الفرس " (الساسانيين)" علي الروم (البيزنطيين)

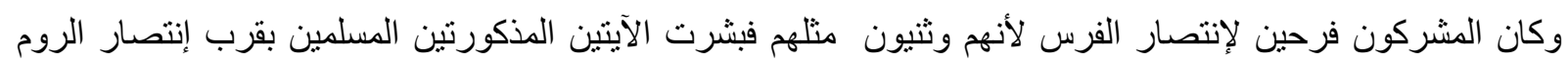
علي الفرس(9)، وقد جاء هذا الجزء من الآيتين المذكورنين من سورة الروم لأول مرة علي نقود الخليفة العباسي المأمون

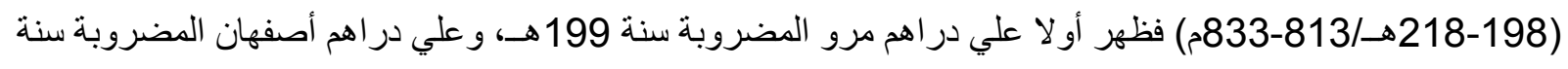

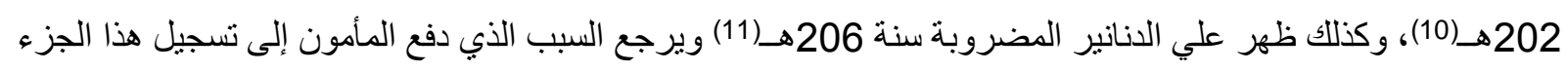

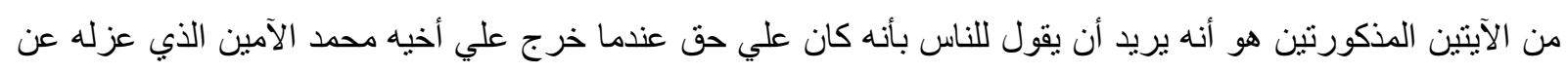

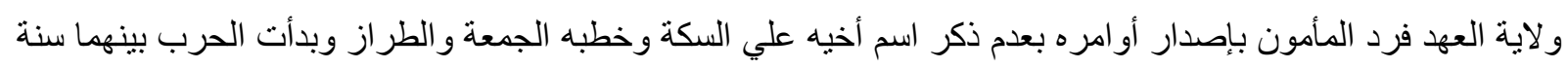

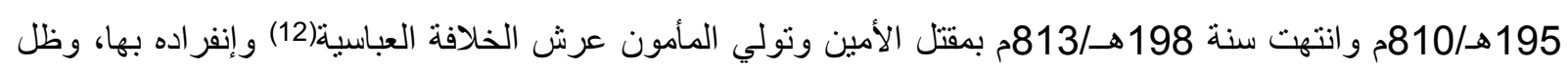
هذا النص القرآني من سورة الروم يسجل علي نقود مختلف الدول الإسلامية حنى نهاية القرن الثامن الهجري/ الرابع عشر الثر الميلادي علي أقل تقدير . ونقش بالسطر الأول بكتابات مركز الظهر كلمة "له " وفي السطر الثاني" محمد "وبالسطر الثالث " رسول الله " وجاء

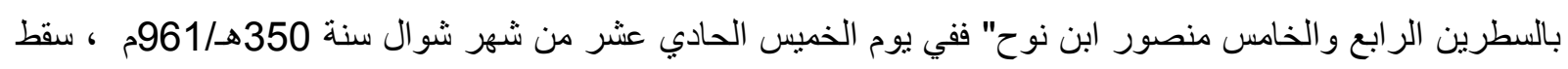


الأمير عبد الملك بن نوح صاحب خر اسان من فوق فرسه علي الأرض فمات(13)، وبعد ذللك قام الوزير أبو محمد بن محمد

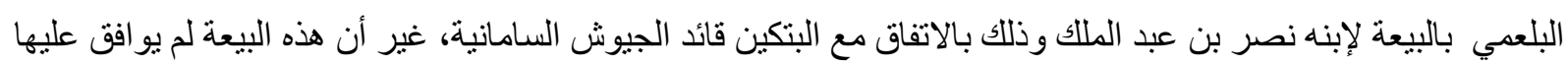

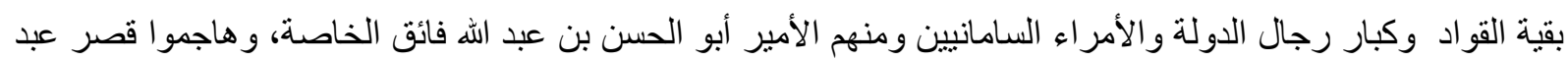

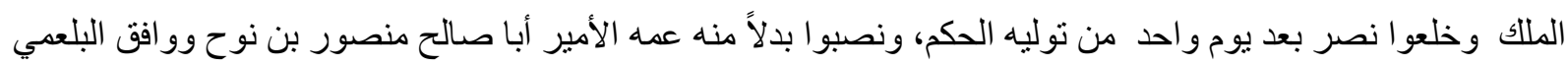

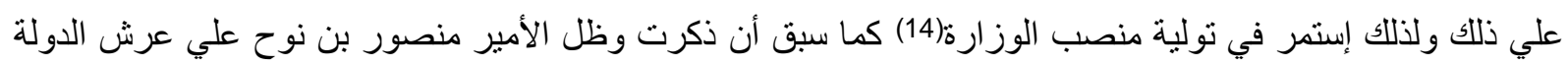

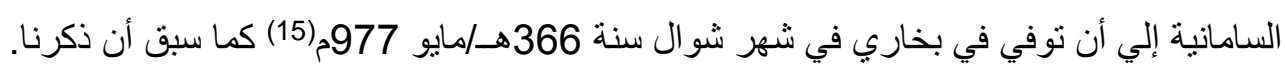

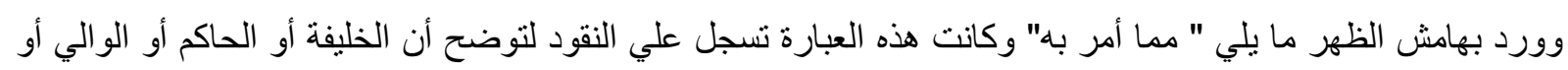
العامل أو الأمير هو صاحب الحق في إصدار ها، وقد إحتفظ الخلفاء الأمويين بحق ضرب النقاب النقود الذهبية والفضية بينما

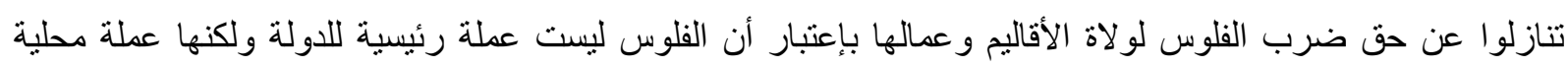
يقتصر تداولها داخل حدود الولاية أو الإقليم الذي ضربت فيه، لذلك تسابق الو لاة و العمال في تسجيل أسمائهم علي الفلوس

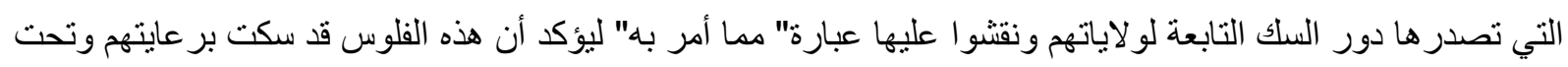

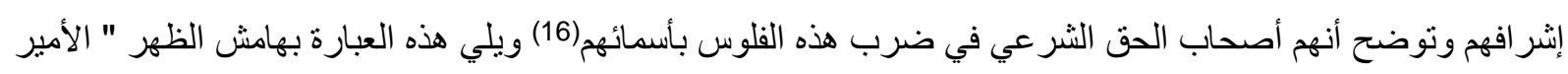

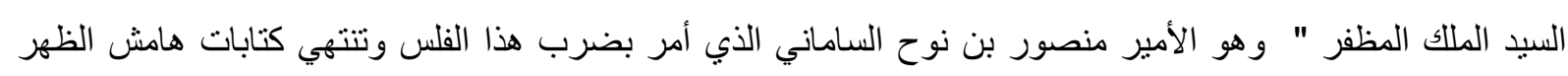

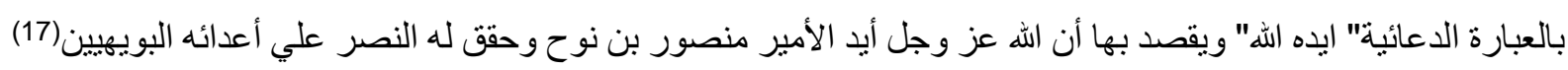

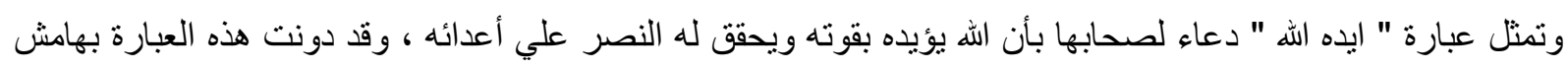

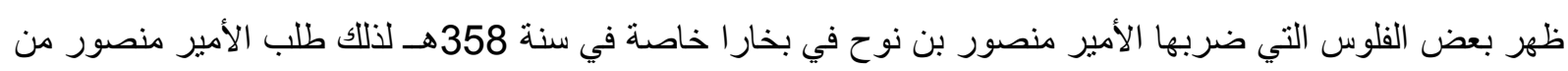
الله النصر والتأييد في حروبه التي خاضها ضد أعدائه خاصة البويهيين أن الحروب إستمرت بين فين السامانيين و البو يهيين حول إقليم الري وآل أمر هذه الحروب أيضا إلي عقد صلح بين الأمير الساماني منصور بن نوح و الأمير البويهي ركن الدولة علي أن يدفع الأمير ركن الدولة في كل سنة مائة ألف دينار ويحمل إبنه عضد الدولة إلي الأمير الساماني خمسين

ألف دينار (18).

وقد وصلنا العديد من الفلوس السامانية التي تحمل إسم الأمير منصور بن نوح و المضروبة في بخار اسنة 358هـ وتحمل

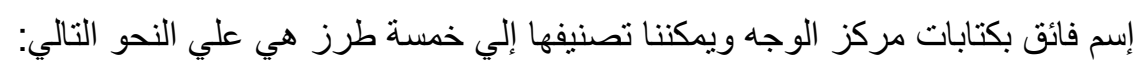

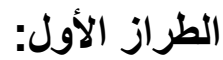

يثتمل هذا الطر از على كتابات مركزية في خمسة أسطر متو ازية تدور حولها كتابات في هامش واحد وذلك بكل من الوجه

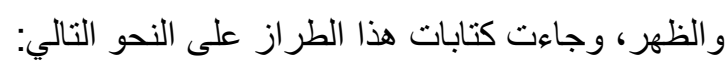

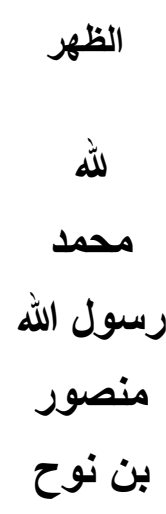

هامش: مما امر به الامير السيد الملك المظقر

ايده الله

الوجه

\section{فائق}

لا إله الا

الله وحده

لاشريك لله

الخاصة

هامش: بسم الله ضرب هذا الفلس ببخارا على يدي ابي بكر سنة ثمان وخمسين وثلثمائة 
وينفرد هذا الطر از عن الطرز الأربعة الأخرى بوجود إسم فائق أعلي كتابات مركز الوجه ولقبه وهو الخاصة أسفل الكتابات

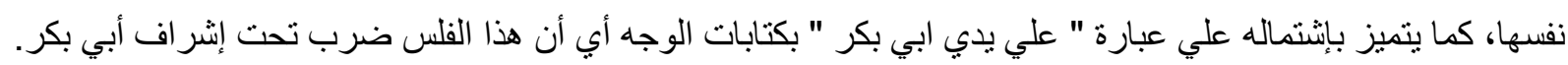
وقد وصلنا من هذا الطر از-علي حد علمنا وفي ضوء ما وصلنا- سنة نماذج من الفلوس التي ضربها منصور بن نوح في بخار اسنة 358هـ وتحمل إسم فائق أعلي كتابات مركز الوجه وسبق نشرها، النموذج الأول محفوظ بدار الكتب المصرية

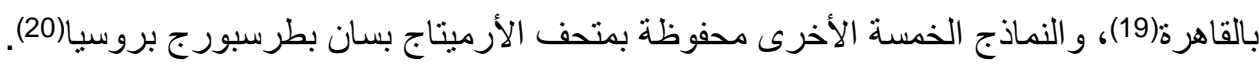

الطراز الثاني: يشبه الطر از الأول في شكله العام ونصوص كتاباته وترتيبها عدا كتابات هامش الوجه التي جاءت هنا علي النحو التالي :

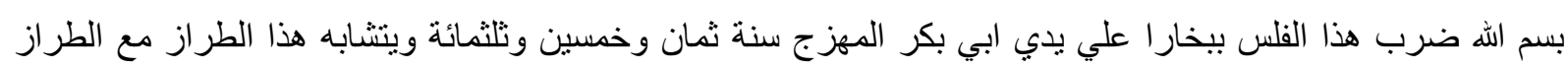
الأول في كتابات مركز الوجه عدا أن هذا الطر از يتميز بإنتماله علي إسم أو بكر المهزج كاملا هكذا: علي يدي أبي بكر

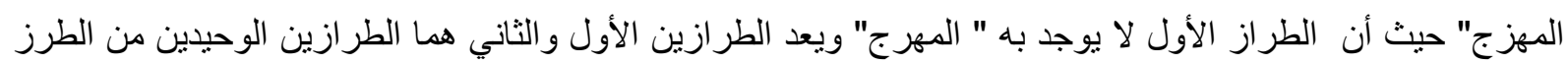
الخمسة الذين يحملان إسم الآمر بضرب الفلس ببخار اسنة 358هـ بصيغة " علي يدي ابي بكر" في الطر از الأول وبصيغة

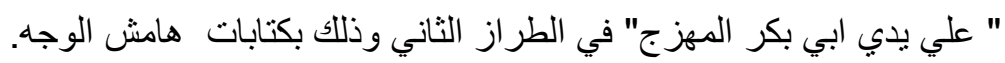
وينسب لهذا الطراز نموذج(21)نشره لين بول محفوظ بالمتحف البريطاني بلندن.

الطراز الثالث: يشبه الطراز الأول في الثكل العام وكتابات الظهر وترتيبها ولكنه يختلف عنه في كتابات الوجه وجاءت كتابات هذا الطر از علي النحو النتالي:

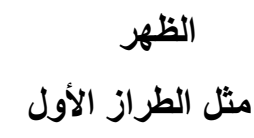

هامش: بسم الله ضرب هذا القلس ببخارا سنة ثمان وخمسين وثلثمائة ويختلف هذا الطراز عن الطرز الأربعة الأخرى وهي الأول و الثاني و الر ابع و الخامس بوجود وإسم إسم فائق أعلى كتابات مركز الوجه و إسم بكر أسفل كتابات مركز الوجه أيضا معاً. وينسب لهذا الطر از نموذجين سبق نشر هما، النموذج الأول محفوظ بدار الكتب المصرية بالقاهرة(22) و النموذج الثاني

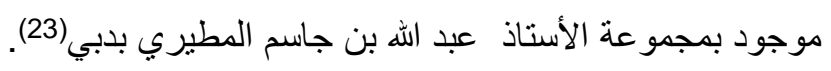




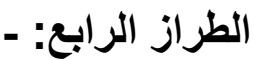
يشبه الطر از الثاني في الثكل العام وكتابات الظهر. ووردت كتاباته على النحو التالي: | مثل الطراز الاول

الوجه

فائق

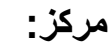

لا إله الا

الله وحده

لاشريك لله

المهززج

هامش: مثل هامش الطراز الثالث ينفرد هذا الطر از عن الطرز الأربعة الأخرى وهي الأول و الثاني و الثالث و الخامس بوجود إسم فائق بأعلي كتابات مركز الوجه بالسطر الأول ووجود إسم المهزج أسفل نفس الكتابات بالسطر الخامس، وينتمي لهذا الطراز فلسين محفوظين بمتحف وله الأرميناج بروسيا(24).

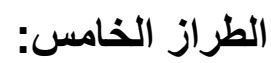
ويتميز هذا الطراز بأنه يوجد بمركز الوجه شكل يشبه المنلث قاعدته لأعلي ور أسه أسفل وبداخل المثلث إسم فائق وأعلي

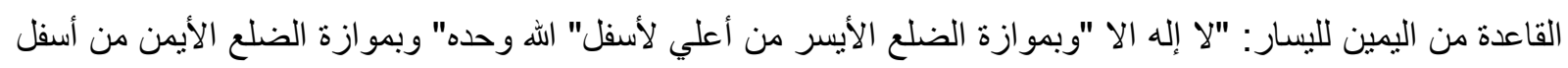

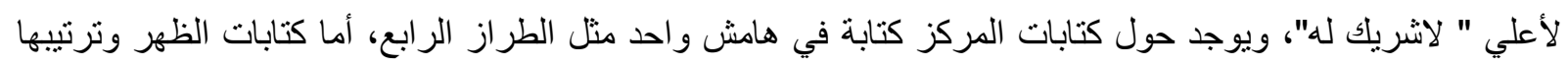
فهي واحدة في الطرز الخمسة. ويختلف هذا الطر از عن الطرز الأربعة السابقة بوجود مثلث بمركز الوجه بداخله اسم فائق.

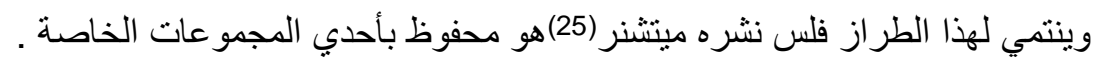
وبمقارنة الفلس موضوع البحث بفلوس الطرز الخمسة التي ضربها الأمبر منصور بن نوح بيخار سنة 358هـ وتحمل

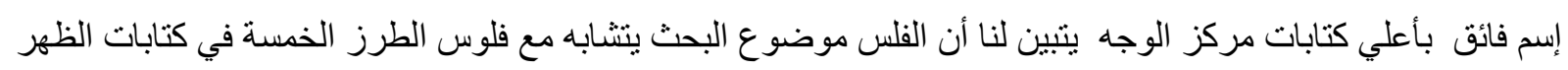
وترتيبها ولكن الفلس موضوع البحث يختلف عن فلوس الطرز الخمسة في كتابات الوجه فقط حيث أن وجه الطرز الخمسة

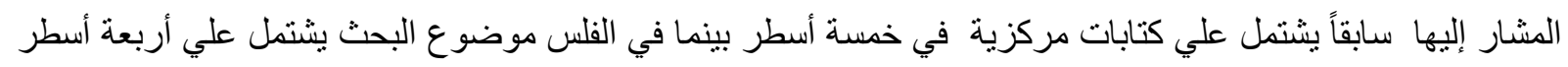
فقط أعلاها بالسطر الأول إسم فائق فقط ولا توجد أسماء أسفل هذه الكتابات المركزية ، كذلك فأنه يحيط بكتابات مركز وجه الطرز الخمسة هامش كتابي واحد ، بينما في الفلس موضوع البحث يوجد هامثين كتابيين حول كتابات المركز،

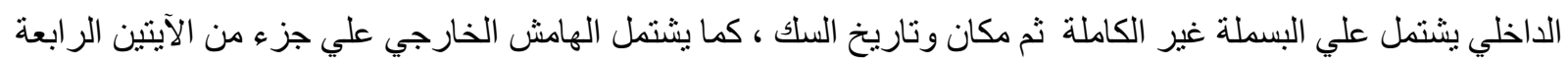
و الخامسة من سورة الروم ونصه " لله الامر من قبل ومن بعد ويومئذ يفرح المؤمنون بنصر الله " وهذا الجزء من الآيتين

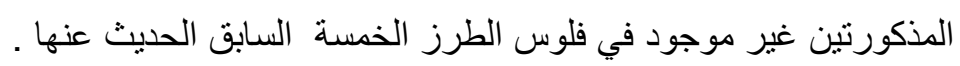
ومن الجدير بالذكر أن الطراز الخامس هو فير الطرز الوحيد من الطرز الخمسة الذي يشتمل علي إسم فائق فقط بكتابات مركز الوجه و هو بذللك يشبه الفلس الموضوع ولكن وضع إسم فائق يختلف فيهما حيث جاء في الطراز الخامس داخل 
مثلث وحوله شهادة التوحيد كاملة بينما جاء إسم فائق أعلى كتابات مركز الوجه وتتفق فلوس الطراز الخامس مع الفلس موضوع البحث بأنه لا بوجد بكتابات مركز الوجه أسماء أخرى غير فيد " فائق" فقط.

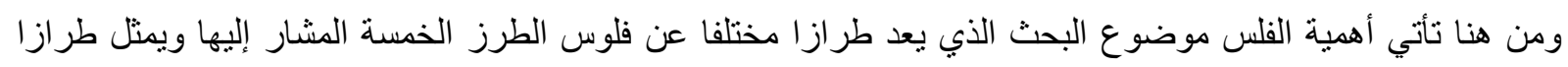
جديدا غير معروف من قبل خاصة أن هذا الفلس لم يسبق نشره أو دراسته من قبل، كما لم ينشر مثيل له فهو طر ازئ جديد

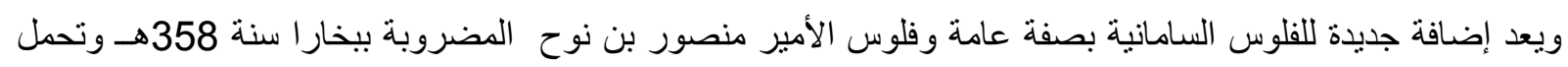

إسم فائق بصفة خاصة. المراجع والهوامش والتعليقات: - (الن

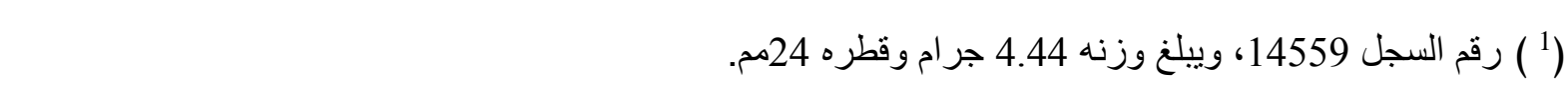

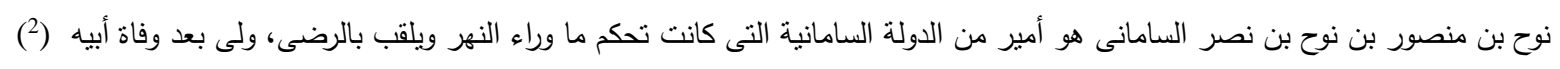

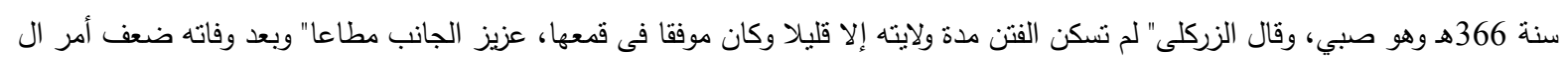

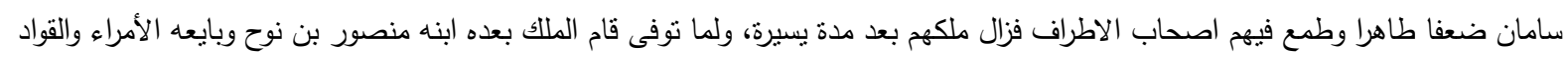

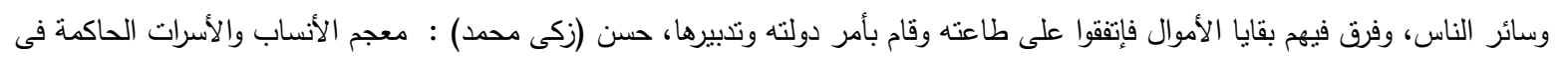

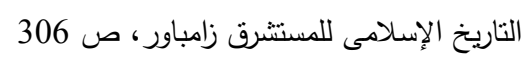

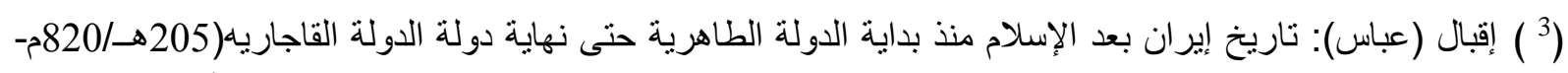

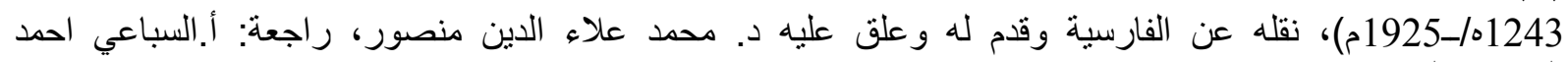

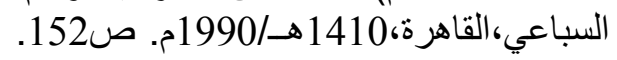
'Iqbal (ebas): Tarikh 'iiran baed al'islam mundh bidayat aldawlat alttahiriat hataa nihayat dawlat aldawlat alqajaryh(205h/820m-1243h/1925m), naqlih ean alfarisiat waqadam lah waealaq ealayh d. muhamad eala' aldiyn mansur, rajiet: a.alsbaey 'ahmad alsabaei,alqahrt,1410h/1990.

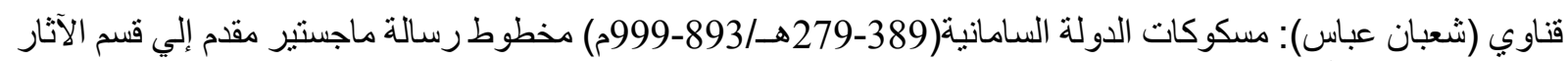

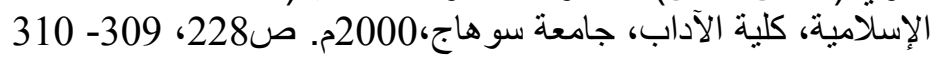
Qanawi (sheiban eibas): maskuakat aldawlat alsaamanit(279-389h/893-999ma) makhtawt risalat majsatayr muqadim 'iilay qism alathar al'iislamiat, kuliyat aladab, jamieat suhaj,2000.

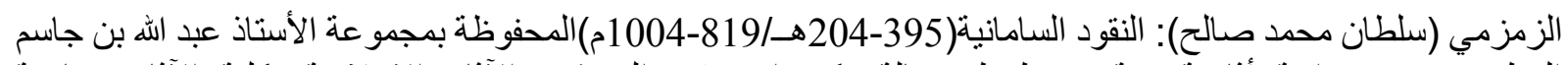

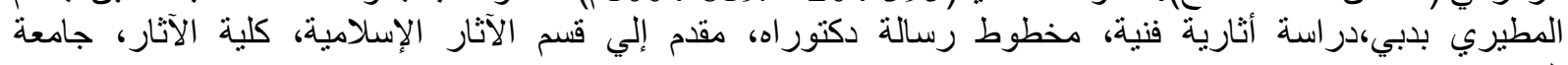

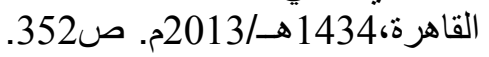

Alzamzami (slutan muhamad salh): alnuqud alsaamanit(204-395ha/819-1004mi)almahfuzat bimajmueat al'ustadh eabd allah bin jasim almutayri badibia,dirasat 'athariat faniat, makhtawt risalat dukturah, muqadim 'iilay qism alathar al'iislamiati, kuliyat alathar, jamieat alqahirat, $1434 \mathrm{ha} / 2013$.

$$
\text { ( ) قاز ان (وليم): المسكوكات الإسلامية ،بيروت، 1404هـ/1983م. 350- 359. }
$$

Qazan (wlym): almaskukat al'iislamiat ,bayruut, 1404ha/1983.

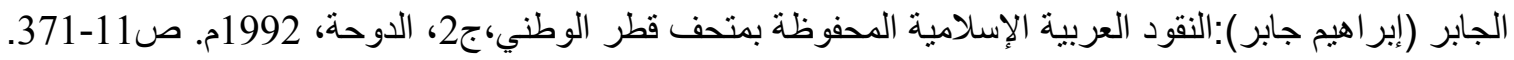
Aljabir ('iibirahim jabir):alnuqud alearabiat al'iislamiat almahfuzat bimutahaf qatar alwatani,j2, aldawhat, 1992.

Alqysyu (nahd eabd alrazq): alnqwd fi aleiraq,bighdad,2002,

$$
\text { القيسي (ناهض عبد الرازق): النقود في العر اق،بغداد،2002م. }
$$

النشرعان (نايف) : التعدين وسك النقود في الحجاز ونجد وتهامة في العصرين الأموي والعباسي،1اط1،

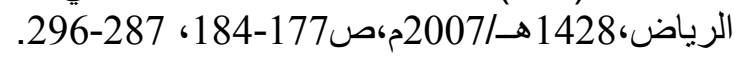


Alshuruean (nayf) : altaedin wask alnuqud fi alhijaz wanajd watahamat fi aleisrin al'umawii waleabasi,t1, alriyad,1428ha/2007m,s177-184, 287-296.

Norman D.Nicol,Raafat M.el-Nabrawy,Jere L Bacharach: Catalog of The Islamic Coins ,Glass Weights ,Dies and Medals In The Egyptian National Library Cairo California U.S.A,1982,P.2 Nos.44-45, Balog,Paul: The coinage of The Malmluk sultans, of Egypt and Syria, New York,1964, Balog,Paul: The coinage of the Ayyubids,London,1980 ؛Lane -poole, Stanley, catalogue of The Oriental Coins in the British Museum, vols.1-10,London,1875-1890, Lavoix,Henri: Catalogue de Monnaies Musulim= =manes de La Bibliothque Nationale, 3 vols,Paris, $1887-1890$.

$\left(^{5}\right)(4)$ Walker ,John: A.Catalouge of Muhammadan Coins In The British Museum,Vol I,Arab -Sassanian, London, 1941,p.3,No.2

(6 ) النرشخي: ( ابو بكر محمد بن جعفر النرشخي ): تاريخ بخاري، نرجمة امين عبد المجيد بدوي ونصر الله مبشر

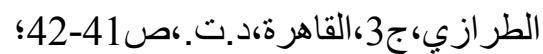

Alnarshukhi: ( 'abu bakr muhamad bin jaefar alnurshkhi ): tarikh bikhari, tarjamat 'amin eabd almajid bidawi wanasr allah mubashir altarazi,ja3,aulqahirat,d.t.,s41-42;

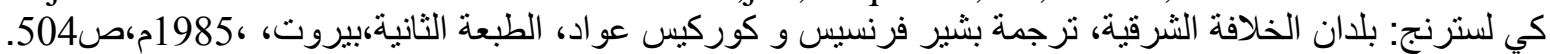
kay lastarnj: buldan alkhilafat alsharqiati, tarjamat bashir fransis w kurkis eawad, altibeat althaaniat,birawt, ,1985m,s504.

(7 ) الاصطخري : ( أبو إسحاق إبراهيم بن محمد الفارسي الاصطخري ،ت 346هـ ):مسالك الممالك، ليدن مطبعة

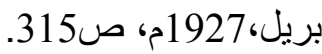

Alaistikhriu : ( 'abu 'iishaq 'iibrahim bin muhamad alfarisi alaistakhri ,t 346h ):misalik almumaliku, lidan mutbaeat biril,1927m, sa315.

(8) ) أرمنيوس (فامبري) : تاريخ بخاري منذ أقدم العصور حتى الوقت الحاضر، ترجمه د. أحمد محمود الساداتي ،القاهرة

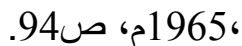

'Urmunyus (fambry) : tarikh bikhari mundh 'aqdam aleusur hataa alwaqt alhadiri, tarjamah da. 'ahmad mahmud alsaadati ,alqahirat ,1965ma, s94.

(9 ) القرطبي:( أبو عبد الله محمد بن احمد الأنصاري): الجامع لإحكام القرآن، ط3، القاهرة،1387هـ/1967م،ج14،ص11-

Alqartaby:( 'abu eabd allah muhamad bin 'ahmad al'ansari): aljamie li'iihkam alqurani, t3, alqahirt,1387ha/1967ma,j14,sa1-7.

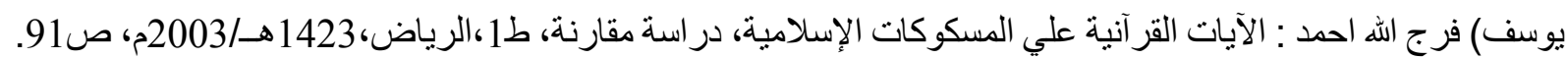
Yusif (faraj allah 'ahmad) : alayat alquraniat ealia almaskukat al'iislamiatu, dirasat muqarinat, ta1,alriyad,1423ha/2003mi, s91.

$$
\text { (10 ) شما (سمير) : أحداث عصر المأمون كما ترويها النقود، الاردن، 1415هـ/1995م، ص225. }
$$

Shamaa (Samir) : 'ahdath easr almamun kama tarawiyha alnaqud, alardun, 1415ha/1995m, s225.

NesselmanG.H.F: Die Orientalischen Munzen des A kadeemischen munzcabinet Inkonigsbsrg Leipzig,1858,p.65 ,Miles,G.C: The Numismatic History of Rayy.The ANS.New York,1938,p.103.

$$
\text { شما (سمير) : أحداث عصر المأمون، ص 336-337. }
$$

Shamaa (Samir) : 'ahdath easr almamun, s 336-337.

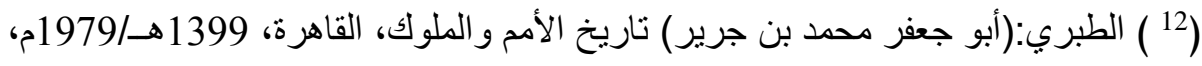

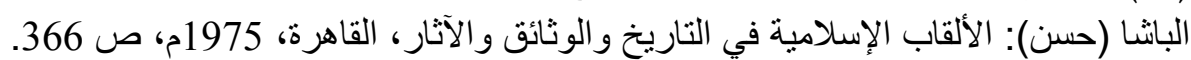

Altaburay:('abu jaefar muhamad bin jarira) tarikh al'umam walmuluik, alqahirat, 1399ha/1979ma, 
Albasha( hasan): al'alqab al'iislamiat fi alttarikh walwathayiq walathar, alqahirat, 1975m, s 366.

$$
\text { يوسف (فرج اله احمد): الآيات القرآنية علي المسكوكات الإسلامية، ص } 91 .
$$

Yusuf (fraj allah ahmada): alayat alquraniat ealia almaskukat al'iislamiat, s 91.

(13 ) ابن الأثير: (أبي الحسن علي بن إبي الكرم محمد الثيباني، ت 630هـ/232م):-الكامل في التاريخ، مجلد 7، ط1،

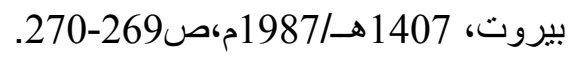

Abn al'athir: (abi alhasan eali bin 'iiby alkaram muhamad alshiybani, t 630h/1232m):-alakamil fi altaarikh, mujalad 7, t1, bayruut, 1407ha/1987m,s269-270.

( إقبال (عباس) : تاريخ إيران،ص152.

'Iqbal (ebas) : tarikh 'iiran,sa152.

$$
\text { (15 ) (15ن الأثثر : الكامل في التاريخ،ج7،ص367. }
$$

Abn al'athir : alkamil fi altaarikh,j7,s367.

'Iqbal (ebas) : tarikh 'iiran,sa154

إقبال (عباس) : تاريخ إيران،ص 154.

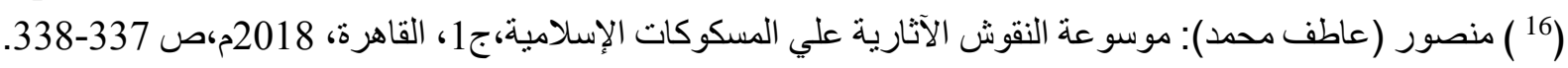

Mansur (eatif muhmd): mawsueat alnuqush alathariat ealia almaskukat al'iislamiat,j1, alqahirut, 2018m,s 337-338.

Alzamzimi (Slatan) : alnuqud alsaamaniat: sa352.

$$
\text { ( ) الزمزمي (سلطان) : النقود السامانية: ص352. }
$$

$$
\text { ( إقبال (عباس) : تاريخ إيران، ص154؛ }
$$

'iqbal (ebas) : tarikh 'iiran, sa154;

(حسن) إبر اهيم حسن: تاريخ الإسلام السياسي و الديني و الثقافي و الاجتماعي،ج3، القاهرة، 1411هـ/19911، ص 86؛ (Hasan) Ibrahim Hasan: tarikh al'islam alsiyasii waldiynii walthaqafii walaijtimaei,j3, alqahiratu, 1411h/1991m, s 86;

منصور (عاطف محمد) : موسو عة النقوش الآثارية، ج1، ص 226-225-226. Munsur(Eatif Muhmd) : mawsueat alnuqush alathariat, ja1, s 225-226.

(19) Norman Nicol and others: op.cit,p10,No 409.

$$
\text { (20 ) قناوي (شعبان) : مسكوكات الدولة السامانية ،ص 309-310، كتالوج الرسالة أرقام 2569-309-310, }
$$

Qanawi (sheibana) : maskukat aldawlat alsaamaniat ,s 309-310, kitalwj alrisalat 'arqam 25692574.

Markov,A: Inventary Katalog MusulmansKikh Monet Imperatorskogo Ermitaga,Cpb,1896,.pp1048-1050.

$$
\text { (21 ) فناوي (شعبان ): مسكوكات الدولة السامانبة ،كتالوج رقم 2559، لوحة 79،ص 310. }
$$

Qanawi (sheabban ): maskukat aldawlat alssamaniat ,kitaluj raqm 2559, lawhatan 79,s 310.

, Lane -poole, Stanley, catalogue of The Oriental Coins in the British Museum, vol.II,London,1896,p.110,No.409.

$$
\text { ( } 22 \text { ) قناوي (شعبان ): مسكوكات الدولة السامانية ،كتالوج رقم 2559، }
$$

Qanawi (sheabban ): maskukat aldawlat alssamaniat, kitaluj raqm 2559.

Norman Nicol and others: op.cit, ,No 4555.pl .XX

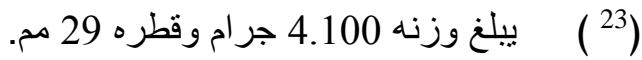

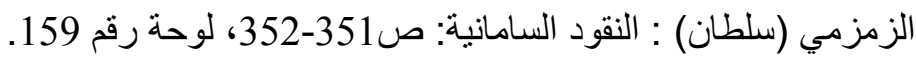

Alzamzimi (sltan) : alnuqud alssamaniat: s351-352, lawhatan raqm 159. 


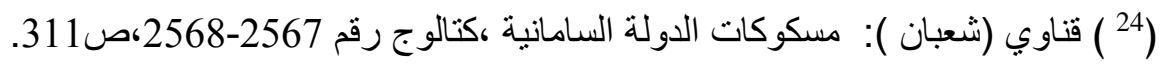
Qanawi (sheibaan ): maskukat aldawlat alsaamaniat ,kataluj raqm 2567-2568,sa311. Markov, op.cit,p.157,Nos1041-1042.

$$
\text { ( }{ }^{25} \text { قناوي (شعبان ): مسكوكات الدولة السامانية، ص 312-312، كتالوج رقم 2562،لوحة 79. }
$$

Qanawi (sheibaan ): maskukat aldawlat alsaamaniat, s 312-313, kitaluj raqm 2562,lawhat 79. Mitchiner,M:The World of Islam, oriental coins and their values , London , 1966, p.139,No.705.
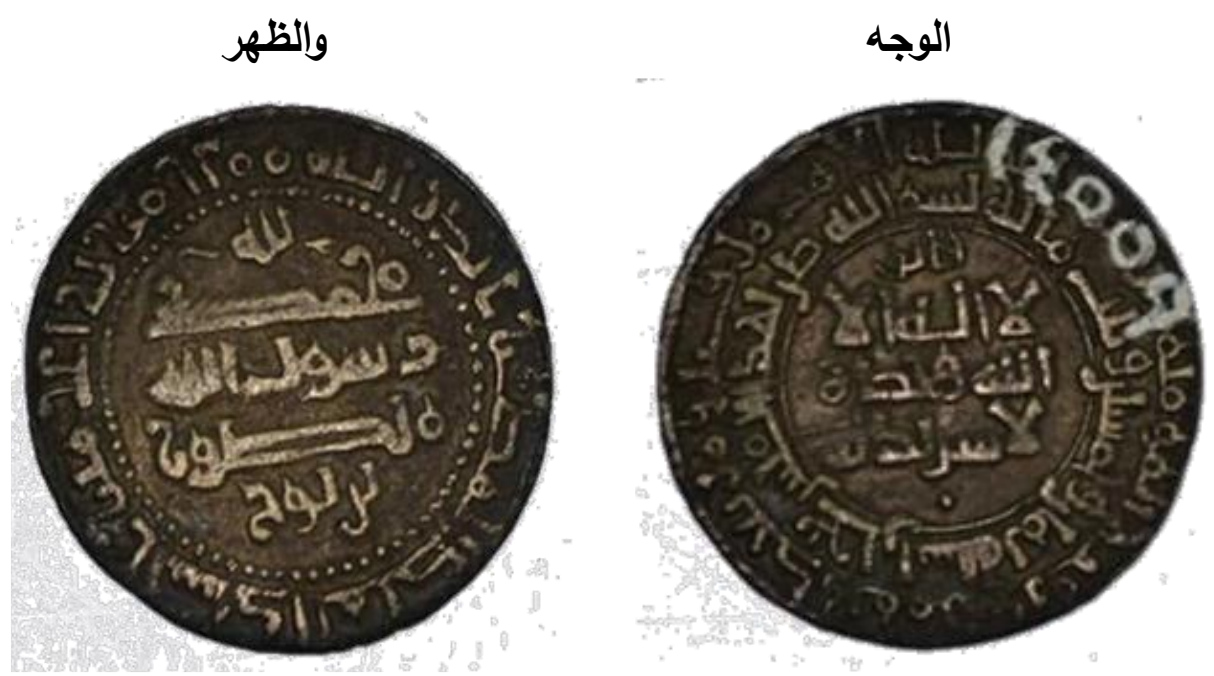

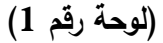

$$
\begin{aligned}
& \text { فلس ساماني للأمير منصور بن نوح ضرب بخار سنة 358هـ } \\
& \text { محفوظ بمتحف الفن الإسلامي بالقاهرة }
\end{aligned}
$$

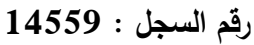
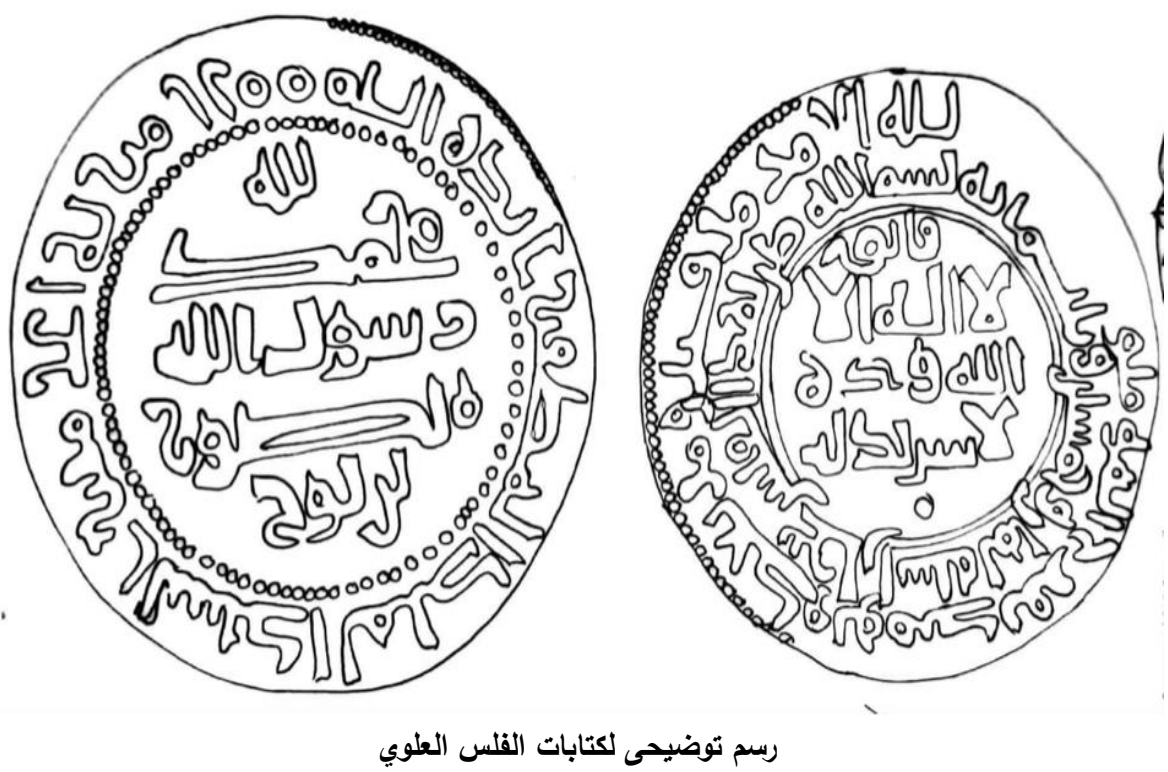